\title{
IMPACT OF MIGRATION ON EPIDEMIOLOGICAL DYNAMICS WITH SATURATED INCIDENCE RATE
}

\author{
ALI AL-QAHTANI, SHABAN ALY, AND FATMA HUSSIEN
}

Received 14 April, 2017

\begin{abstract}
Recently an SIS epidemic reaction-diffusion model with Neumann (or no-flux) boundary condition has been proposed and studied by several authors to understand the dynamics of disease transmission in a spatially heterogeneous environment in which the individuals are subject to a random movement. In this paper an SIS epidemiological model with saturated incidence rate is proposed to describe the dynamics of disease spread among identical patches due to population migration. First the stability conditions for the endemic equilibrium for the corresponding kinetic system and reaction-diffusion system without diffusion are analyzed and proved. Moreover, we prove that at a critical value of the bifurcation parameter the positive endemic equilibrium becomes linear non-constant stationary solutions only when diffusion also plays a role in the reaction-diffusion system, which shows that the strong effects of diffusion on the Turing instability. Numerical simulations are provided to illustrate and extend the theoretical results.
\end{abstract}

2010 Mathematics Subject Classification: 34C23; 35K57; 92D25; 92D30

Keywords: SIS Epidemic Model, Patchy space, Turing instability

\section{THE Model}

Infectious diseases have tremendous influence on human life and will bring huge panic and disaster to mankind once out of control. Every year millions of human beings suffer from or die of various infectious diseases. In order to predict the spreading of infectious diseases, many epidemic models have been proposed and analyzed in recent years $([6,10,19,20,22])$. In epidemiology, mathematical models have been an important method in analysing the spread and control of infectious diseases qualitatively and quantitatively. More recently, many studies have provided that spatial epidemic model is an appropriate tool for investigating fundamental mechanism of complex spatiotemporal epidemic dynamics ([3,11]). In these studies, reaction diffusion equations have been intensively used to describe spatiotemporal dynamics and pattern formation in the spatial epidemic model, starting with the pioneer work of Turing. The Turing instability ([18]) has been extensively investigated for biological and chemical processes. Moreover, pattern formation from the Turing instability in 
nonlinear complex systems is actively investigated in the fields such as social networks, molecular computing, embryology and elsewhere in biology and chemistry $([4,5,9,12])$.

Continuous models, usually in the form of nonlinear ordinary differential equations (NODE), have formed a large part of the traditional mathematical epidemiology literature. In such models, the classical assumptions are that the total population is divided into any number of classes according to their epidemiological status, and that the transmission of the infection in the population is modelled by incidence terms. Some infectious diseases do not confer immunity. Such infections do not have a recovered state and individuals become susceptible again after infection. Diseases such as tuberculosis, meningitis, sexually transmitted diseases or bacterial infections and gonorrhea exhibit this phenomenon $([4,9,12])$. This type of disease can be modelled by the SIS type. Many forms are possible for the incidence term in epidemic models, the most common are the simple mass action and standard incidence terms. $\mathrm{Li}$ et al. ([8]) studied an $S I S$ model with bilinear incidence rate $\beta S I$ and treatment. In this paper, we consider saturation incidence rate and assume the force of infection is in this version $\frac{\beta S^{n} I^{m}}{1+c S}$ which is saturated with the susceptible. In this paper we demonstrate how a mathematical model can describe epidemiological phenomena and how can we use such a model to analyze endemic states and help eradicate disease. A model was set up using a system of nonlinear ordinary differential equations and show how the Turing phenomenon looks like in this ODE setting.

We consider a $S I S$ type of disease transmission. The population is divided into two classes: susceptible individuals and infectious individuals. Susceptible individuals become infective after contact with infective individuals. Infective individuals return to susceptible class when they are recovered i.e. no immunity is conferred by going trough the disease.

However, in nature, the tendency of the susceptible would be to keep away from the infected for the reason that the susceptible have ability to recognize the infected group and move away from them (cf. [7, 14-16]). Diseases such as tuberculosis, meningitis, bacterial infections and sexual diseases can be easily transmitted from one country (regions, cities or patch) to other countries (regions, cities or patch)(cf. $[1,2,17])$. Thus, it is important to consider the impact of migration on spread of a disease. Our interest is to study a $S I S$ epidemiological model in patchy space in which the per capita migration rate of infective specie is influenced only by its own density (self-migration), and the per capita migration rate of susceptible specie is influenced not only by its own density but also to the density of the other one (cross-migration).

To formulate the SIS epidemic diffusion model in the following subsections, some assumptions and parameters are specified as follows.

The susceptible individuals and the infected individuals in city $k$ at time $t$ are denoted as $S_{k}(t)$ and $I_{k}(t)$, respectively $\left(k \in\{1 ; 2\} ; t \in \mathbb{R}_{0}^{+}\right) . \quad 1 \leq m, n \in \mathbb{N}$ are 
constants describing the incidence rate of the disease. $a$ and $b$ are the natural birth rate and the natural death rate, respectively. $\gamma$ is the recovery rate. $\alpha$ is the mortality caused by the disease. $\beta$ is the disease transmission coefficient. $c$ is the saturation factor that measures the inhibitory effect. $d_{i}>0$ are the diffusion coefficients $(i \in$ $\{1 ; 2\}) . \rho \in C^{1}$ are positive functions modeling the cross-diffusion effect.

Hence, the ordinary differential equations (ODE) for the $S I S$ epidemic diffusion model can be formulated as

$$
\begin{aligned}
& \dot{S}_{1}(t)=-\frac{\beta S_{1}^{n} I_{1}^{m}}{1+c S_{1}}-b S_{1}+\gamma I_{1}+a\left(S_{1}+I_{1}\right)+d_{1}\left(\rho\left(I_{2}\right) S_{2}-\rho\left(I_{1}\right) S_{1}\right), \\
& \dot{I}_{1}(t)=\frac{\beta S_{1}^{n} I_{1}^{m}}{1+c S_{1}}-(\alpha+b+\gamma) I_{1}+d_{2}\left(I_{2}-I_{1}\right), \\
& \dot{S}_{2}(t)=-\frac{\beta S_{2}^{n} I_{2}^{m}}{1+c S_{2}}-b S_{2}+\gamma I_{2}+a\left(S_{2}+I_{2}\right)+d_{1}\left(\rho\left(I_{1}\right) S_{1}-\rho\left(I_{2}\right) S_{2}\right), \\
& \dot{I}_{2}(t)=\frac{\beta S_{2}^{n} I_{2}^{m}}{1+c S_{2}}-(\alpha+b+\gamma) I_{2}+d_{2}\left(I_{1}-I_{2}\right) .
\end{aligned}
$$

ODEs (1.1) describes the following dynamics of epidemic diffusion among the population groups. The per capita migration rate of infective specie is influenced only by its own density (self-migration), and the per capita migration rate of susceptible specie is influenced not only by its own density but also to the density of the other one (cross-migration).

\section{Model ANALYSis}

In this section we analyze a simple model of the temporal behavior of an infectious disease which is not extended in space $\left(d_{1}=d_{2}=0\right)$,

$$
\begin{aligned}
& \dot{S}_{1}(t)=-\frac{\beta S_{1}^{n} I_{1}^{m}}{1+c S_{1}}-b S_{1}+\gamma I_{1}+a\left(S_{1}+I_{1}\right), \\
& \dot{I}_{1}(t)=\frac{\beta S_{1}^{n} I_{1}^{m}}{1+c S_{1}}-(\alpha+b+\gamma) I_{1}, \\
& \dot{S}_{2}(t)=-\frac{\beta S_{2}^{n} I_{2}^{m}}{1+c S_{2}}-b S_{2}+\gamma I_{2}+a\left(S_{2}+I_{2}\right), \\
& \dot{I}_{2}(t)=\frac{\beta S_{2}^{n} I_{2}^{m}}{1+c S_{2}}-(\alpha+b+\gamma) I_{2} .
\end{aligned}
$$

Define the new variable $N(t)=S(t)+I(t)$. In each patches, summing the two equations in (2) provides the equation for the total population $\dot{N}(t)=(a-b) N-\alpha I$. 
Thus, the total population size may vary in time, as traditionally assumed. In the absence of a mortality rate caused by the disease, and assuming a greater birth rate than a mortality rate, i.e., $\alpha=0$ and $a>b$, the population will grow indefinitely. Therefore, we could think of the disease as regulating the growth of the population. For this reason, we will assume from here on that $\alpha>a-b>0$. These facts are known substantially from paper of Carrero et al (cf. [3]).

In this case the unique positive equilibrium of (2.1) is

- $(\bar{S}, \bar{I}, \bar{S}, \bar{I})_{1}$ where $m=1$ and

$$
I=g_{1}(S)=\frac{(1+c S)(\alpha+b+\gamma)}{\beta S}, \quad I=g_{2}(S)=\frac{a-b}{\alpha+b-a} \cdot S
$$

resp.

- $(\bar{S}, \bar{I}, \bar{S}, \bar{I})_{m}$ where $1<m \in \mathbb{N}$ are the intersection of the curves

$$
I=h_{1}(S)=\left(\frac{(\alpha+b+\gamma)(1+c S)}{\beta S^{n}}\right)^{\frac{1}{m-1}}, \quad I=h_{2}(S)=\frac{a-b}{\alpha+b-a} \cdot S .
$$

The Jacobian matrix of system (2.1) linearized at $(\bar{S}, \bar{I}, \bar{S}, \bar{I})_{1}$ is

$$
\begin{aligned}
& J(n, 1)= \\
& \left(\begin{array}{cccc}
R & -(\alpha+b-a) & 0 & 0 \\
(a-b) T\left(n-\frac{c \bar{S}}{1+c \bar{S}}\right) & 0 & 0 & 0 \\
0 & 0 & R & -(\alpha+b-a) \\
0 & 0 & (a-b) T\left(n-\frac{c \bar{S}}{1+c \bar{S}}\right) & 0
\end{array}\right)
\end{aligned}
$$

where $T:=\frac{\alpha+b+\gamma}{\alpha+b-a}$ and $R:=(a-b)\left(1-n T+\frac{c \bar{S}}{1+c \bar{S}} \cdot T\right)$.

The equilibrium $(\bar{S}, \bar{I}, \bar{S}, \bar{I})_{1}$ is locally asymptotically stable if the zeroes of the characteristic polynomial

$$
P_{4}(\lambda, n, 1)=\left(P_{2}(\lambda, n, 1)\right)^{2},
$$

have negative real part, where

$$
P_{2}(\lambda, n, 1)=\lambda^{2}-(a-b)\left(1-n T+\frac{c \bar{S}}{1+c \bar{S}} \cdot T\right) \lambda+(a-b)(\alpha+b+\gamma)\left(n-\frac{c \bar{S}}{1+c \bar{S}}\right) .
$$

Because

$$
\operatorname{det}(J(n, 1))=(a-b)(\alpha+b+\gamma)\left(n-\frac{c \bar{S}}{1+c \bar{S}}\right)>0,
$$

the zeroes of the characteristic polynomial (2.3) have negative real part, and the equilibrium point $(\bar{S}, \bar{I}, \bar{S}, \bar{I})_{1}$ is locally asymptotically stable if $n>n^{*}$ and unstable if $n<n^{*}$, where

$$
n^{*}=\frac{\alpha+b-a}{\alpha+b+\gamma}+\frac{c \bar{S}}{1+c \bar{S}} .
$$


The Jacobian matrix of system (2.1) linearized at $(\bar{S}, \bar{I}, \bar{S}, \bar{I})_{m}$ is

$$
J(n, m)=\left(\begin{array}{cccc}
J_{11} & J_{12} & 0 & 0 \\
J_{21} & J_{22} & 0 & 0 \\
0 & 0 & J_{11} & J_{12} \\
0 & 0 & J_{21} & J_{22}
\end{array}\right) .
$$

where

$$
\begin{array}{ll}
J_{11}=(a-b)\left(1-n T+\frac{c \bar{S}}{1+c \bar{S}} \cdot T\right), & J_{12}=-m(\alpha+b+\gamma)+a+\gamma \\
J_{21}=(a-b) T\left(n-\frac{c \bar{S}}{1+c \bar{S}}\right), & J_{22}=(m-1)(\alpha+b+\gamma) .
\end{array}
$$

The characteristic polynomial of $J(n, m)$ is

$$
Q_{\text {kinetic }}(\lambda, n, m)=\left(Q_{2}(\lambda, n, m)\right)^{2}
$$

where

$$
Q_{2}(\lambda, n, m)=\lambda^{2}-\left(J_{11}+J_{22}\right) \lambda+J_{11} \cdot J_{22}-J_{12} \cdot J_{21} .
$$

Because

$$
\begin{aligned}
J_{11}+J_{22}= & (a-b)\left(1-n T+\frac{c \bar{S}}{1+c \bar{S}} \cdot T\right)+(m-1)(\alpha+b+\gamma), \\
J_{11} \cdot J_{22}-J_{12} \cdot J_{21}= & (a-b)\left(1-n T+\frac{c \bar{S}}{1+c \bar{S}} \cdot T\right) \cdot(m-1)(\alpha+b+\gamma) \\
& +[m(\alpha+b+\gamma)-a-\gamma] \cdot(a-b) T\left(n-\frac{c \bar{S}}{1+c \bar{S}}\right) \\
= & (a-b)(\alpha+b+\gamma)\left[(m-1)+\left(n-\frac{c \bar{S}}{1+c \bar{S}}\right)\right]>0
\end{aligned}
$$

the equilibrium point $(\bar{S}, \bar{I}, \bar{S}, \bar{I})_{m}$ is asymptotically stable if $n>n^{*}$ (see Figure 1) and unstable if $n<n^{*}$, where

$$
n^{*}=(m-1) \frac{\alpha+b-a}{a-b}+\frac{\alpha+b-a}{\alpha+b+\gamma}+\frac{c \bar{S}}{1+c \bar{S}} .
$$

Note: The assumption comes from the fact that the total population is described by $N^{\prime}(t)=(a-b) N-\alpha I$. Therefore, in order for the disease to have a regulatory effect on the population it is biologically reasonable to assume $\alpha>a-b>0$.

\section{THE IMPACT OF A SELF-MIGRATION}

In this Section, we model the spatial spread as a diffusive process, where both classes have diffusion coefficient. The migration rate of each individuals 

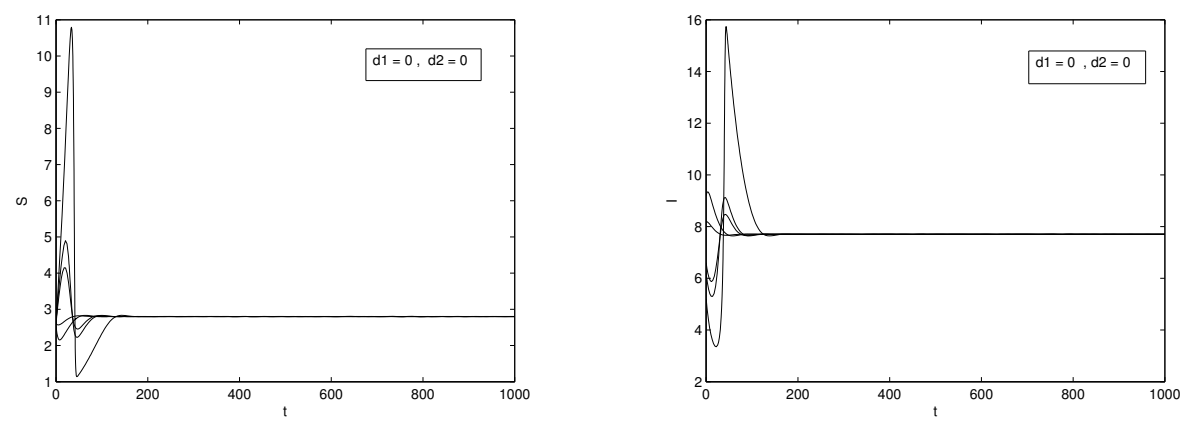

FIGURE 1. The unique endemic solution without diffusion $\left(d_{1}=\right.$ $\left.d_{2}=0\right)$ at $a=0.05, \quad b=0.006, \alpha=0.06, \quad \beta=0.0056$, $\gamma=0.04, \quad c=0.05, \quad m=2, \quad n=1 ; \quad$ is $(\bar{S}, \bar{I}, \bar{S}, \bar{I})_{m}=$ $(2.8013,7.7036,2.8013,7.7036)$ which is stable. (Figure produced by applying MATLAB.)

is influenced only by its own density. The model can be written as:

$$
\begin{aligned}
& \dot{S}_{1}(t)=-\frac{\beta S_{1}^{n} I_{1}^{m}}{1+c S_{1}}-b S_{1}+\gamma I_{1}+a\left(S_{1}+I_{1}\right)+d_{1}\left(S_{2}-S_{1}\right), \\
& \dot{I}_{1}(t)=\frac{\beta S_{1}^{n} I_{1}^{m}}{1+c S_{1}}-(\alpha+b+\gamma) I_{1}+d_{2}\left(I_{2}-I_{1}\right), \\
& \dot{S}_{2}(t)=-\frac{\beta S_{2}^{n} I_{2}^{m}}{1+c S_{2}}-b S_{2}+\gamma I_{2}+a\left(S_{2}+I_{2}\right)+d_{1}\left(S_{1}-S_{2}\right), \\
& \dot{I}_{2}(t)=\frac{\beta S_{2}^{n} I_{2}^{m}}{1+c S_{2}}-(\alpha+b+\gamma) I_{2}+d_{2}\left(I_{1}-I_{2}\right) .
\end{aligned}
$$

Definition 1. The equilibrium $(\bar{S}, \bar{I}, \bar{S}, \bar{I})_{m}$ of (1.1) is said to be diffusionally (Turing) unstable if it is an asymptotically stable of (2.1) but it is unstable with respect to (3.1) (cf. [4-8]).

The Jacobian matrix of the system (3.1) linearized at the endemic equilibria $(\bar{S}, \bar{I}, \bar{S}, \bar{I})_{m}$ given by

$$
J\left(d_{1}, d_{2}\right)=\left(\begin{array}{cccc}
J_{11}-d_{1} & J_{12} & d_{1} & 0 \\
J_{21} & J_{22}-d_{2} & 0 & d_{2} \\
d_{1} & 0 & J_{11}-d_{1} & J_{12} \\
0 & d_{2} & J_{21} & J_{22}-d_{2}
\end{array}\right) .
$$


In order to have Turing instability of the system (3.1), the characteristic polynomial

$\operatorname{det}\left(J\left(d_{1}, d_{2}\right)-\lambda I\right)=Q_{2}(\lambda, n, m)\left[\lambda^{2}-\operatorname{trace}\left(J\left(d_{1}, d_{2}\right)\right)+\operatorname{det}\left(J\left(d_{1}, d_{2}\right)\right)\right]$,

must have at least one eigenvalue with positive real part.

The equilibrium $(\bar{S}, \bar{I}, \bar{S}, \bar{I})_{m}$ is diffusionally unstable if and only if the following criteria holds:

- $Q_{2}(\lambda, n, m)$ has roots with negative real parts;

- $\operatorname{det}\left(J\left(d_{1}, d_{2}\right)\right)<0$

since in this case trace $\left(J\left(d_{1}, d_{2}\right)\right)=\operatorname{trace}(j(n, m))-2\left(d_{1}+d_{2}\right)<0$. This can prove the following Theorem.

Theorem 1. Assume that $n>n^{*}$ holds. Turing instability occurs if

$$
d_{1}>d_{1_{\text {crit }}}=\frac{\operatorname{det}(J(n, m))-2 d_{2}\left[(a-b)\left(1-n T+\frac{c}{1+c \bar{S}} \cdot T\right)\right]}{2(m-1)(\alpha+b+\gamma)-4 d_{2}}
$$

Proof. Since

$$
\begin{aligned}
\operatorname{det}\left(J\left(d_{1}, d_{2}\right)\right)= & \operatorname{det}(J(n, m))-2 d_{2}\left[(a-b)\left(1-n T+\frac{c \bar{S}}{1+c \bar{S}} \cdot T\right)\right] \\
& -2 d_{1}(m-1)(\alpha+b+\gamma)+4 d_{1} d_{2},
\end{aligned}
$$

therefore (3.3) implies Turing instability.

We apply our analytical approach to the following example and we are looking for conditions which imply Turing instability (diffusion driven instability).

Example 1. Trying to prepare an example comparable to that of [1], we choose $a=0.05, b=0.006, \alpha=0.06, \beta=0.0056, \gamma=0.04, c=0.05$, $m=2, n=1, d_{2}=0.05$.

The unique endemic equilibrium is $(\bar{S}, \bar{I}, \bar{S}, \bar{I})_{m}=(2.8013,7.7036,2.8013$, 7.7036). We consider $d_{1}$ as a bifurcation parameter. In this case at $d_{1 \text { crit }} \cong$ 2.4974 , we have four eigenvalues eigenvalues $\lambda_{i}(i=1,2,3,4)$ such that $\Re\left(\lambda_{i}\right)<0,(i=1,2,3)$ and $\lambda_{4}=0$.

Thus,

- if $d_{1}<d_{1 \text { crit }}$ then $\Re\left(\lambda_{i}\right)<0,(i=1,2,3,4)$ and $(\bar{S}, \bar{I}, \bar{S}, \bar{I})_{m}$ is asymptotically stable;

- if $d_{1}>d_{1 \text { crit }}$ then $\Re\left(\lambda_{i}\right)<0,(i=1,2,3), \lambda_{4}>0$, and $(\bar{S}, \bar{I}, \bar{S}, \bar{I})_{m}$ is unstable. 
Therefore, as $d_{1}$ is increases and passes through $d_{1}=d_{1 \text { crit }}$ then the spatially homogeneous equilibrium loses its stability (see Figure 2). Numerical calculations show that two new spatially non-constant equilibria emerge (see Figure 2).
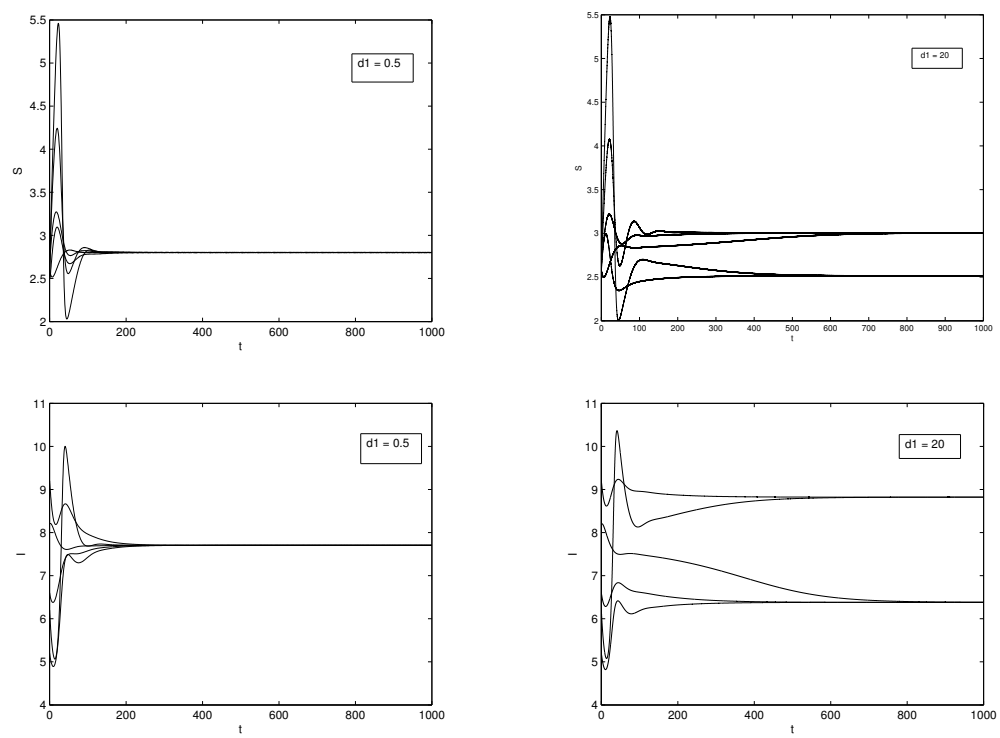

FIGURE 2. Left figures: The solutions $S$ and $I$ before bifurcation at $d_{1}=0.5$; the solution is stable. Right figures: The solutions $S$ and $I$ after bifurcation at $d_{1}=20$; the solutions loss its stability by Turing bifurcation. (Figure produced by applying MATLAB.)

\section{THE IMPACT OF A CROSS-MIGRATION}

In this Section, we model the spatial spread as a diffusive process, where both classes have diffusion coefficient. Rate of infected species is influenced only by its own density, i.e. there is no response to the density of the other one, but the susceptible species is influenced not only by its own but also by the other one's density (cf. [13,21]). The Jacobian matrix of system (1.1) at $(\bar{S}, \bar{I}, \bar{S}, \bar{I})_{m}$ can be written as:

$$
J\left(d_{1}, d_{2}, \rho\right)=\left(\begin{array}{cccc}
J_{11}-d_{1} \rho & J_{12}-d_{1} \rho^{\prime} \bar{S} & d_{1} \rho & d_{1} \rho^{\prime} \bar{S} \\
J_{21} & J_{22}-d_{2} & 0 & d_{2} \\
d_{1} \rho & d_{1} \rho^{\prime} \bar{S} & J_{11}-d_{1} \rho & J_{12}-d_{1} \rho^{\prime} \bar{S} \\
0 & d_{2} & J_{21} & J_{22}-d_{2}
\end{array}\right)
$$


where $\rho$ and $\rho^{\prime}$ are to be taken at $\bar{S}$.

$$
\begin{aligned}
& \operatorname{det}\left(J\left(d_{1}, d_{2}, \rho\right)-\lambda I\right)= \\
& \left|\begin{array}{cccc}
J_{11}-d_{1} \rho-\lambda & J_{12}-d_{1} \rho^{\prime} \bar{S} & d_{1} \rho & d_{1} \rho^{\prime} \bar{S} \\
J_{21} & J_{22}-d_{2}-\lambda & 0 & d_{2} \\
d_{1} \rho & d_{1} \rho^{\prime} \bar{S} & J_{11}-d_{1} \rho-\lambda & J_{12}-d_{1} \rho^{\prime} \bar{S} \\
0 & d_{2} & J_{21} & J_{22}-d_{2}-\lambda
\end{array}\right|
\end{aligned}
$$

Using the properties of determinant $C_{1}^{\prime}=C_{3}+C_{1}, C_{2}^{\prime}=C_{4}+C_{2}$ and $R_{3}^{\prime}=$ $R_{3}-R_{1}, R_{4}^{\prime}=R_{4}-R_{2}$, we get

$$
\begin{aligned}
& \operatorname{det}\left(J\left(d_{1}, d_{2}, \rho\right)-\lambda I\right)= \\
& \left|\begin{array}{cccc}
J_{11}-\lambda & J_{12} & d_{1} \rho & d_{1} \rho^{\prime} \bar{S} \\
J_{21} & J_{22}-\lambda & 0 & d_{2} \\
0 & 0 & J_{11}-2 d_{1} \rho-\lambda & J_{12}-2 d_{1} \rho^{\prime} \bar{S} \\
0 & 0 & J_{21} & J_{22}-2 d_{2}-\lambda
\end{array}\right| .
\end{aligned}
$$

The characteristic polynomial is

$$
Q_{\text {cross }}\left(d_{1}, d_{2}\right)=Q_{2}(\lambda, n, m)\left[\lambda^{2}-\operatorname{trace}\left(J\left(d_{1}, d_{2}, \rho\right)\right) \lambda+\operatorname{det}\left(J\left(d_{1}, d_{2}, \rho\right)\right)\right] .
$$

We know that $Q_{2}(\lambda, n, m)$ has two roots with negative real parts and $(\bar{S}, \bar{I}, \bar{S}, \bar{I})_{m}$ is diffusionally unstable if and only if the following criteria holds:

- $Q_{2}(\lambda, n, m)$ has roots with negative real parts;

- $\operatorname{det}\left(J\left(d_{1}, d_{2}, \rho\right)\right)<0$.

Thus can prove the following Theorem.

Theorem 2. Assume that $n>n^{*}$ holds. Turing instability occurs if

$$
d_{1}>d_{1_{c r i t}}=\frac{\operatorname{det}(J(n, m))-2 d_{2}\left[(a-b)\left(1-n T+\frac{c \bar{S}}{1+c \bar{S}} \cdot T\right)\right]}{2 \rho\left[(m-1)(\alpha+b+\gamma)-2 d_{2}\right]-2 \rho^{\prime} \bar{S}(a-b) T\left(n-\frac{c \bar{S}}{1+c \bar{S}}\right)}
$$

Proof. Since

$$
\operatorname{trace}\left(J\left(d_{1}, d_{2}, \rho\right)\right)=\operatorname{trace}(J(n, m))-2\left(d_{1} \rho+d_{2}\right)<0,
$$


and

$$
\begin{aligned}
& \operatorname{det}\left(J\left(d_{1}, d_{2}, \rho\right)\right)= \\
& =\operatorname{det}(J(n, m))+2 d_{2}\left(2 d_{1} \rho-J_{11}\right)-2 d_{1} \rho\left[J_{22}-\frac{\rho^{\prime}}{\rho} \bar{S} J_{21}\right], \\
& =\operatorname{det}(J(n, m))+2 d_{2}\left[2 d_{1} \rho-(a-b)\left(1-n T+\frac{c \bar{S}}{1+c \bar{S}} \cdot T\right)\right] \\
& \quad-2 d_{1} \rho\left[(m-1)(\alpha+b+\gamma)-\frac{\rho^{\prime}}{\rho} \bar{S}(a-b) T\left(n-\frac{c \bar{S}}{1+c \bar{S}}\right)\right] .
\end{aligned}
$$

therefore (4.4) implies Turing instability.

The equilibrium $(\bar{S}, \bar{I}, \bar{S}, \bar{I})_{m}$ of system (4.4) is asymptotically stable if $\frac{\rho^{\prime}}{\rho}$ is sufficiently large; if $\frac{\rho^{\prime}}{\rho}$ is sufficiently small and either $\rho$ or $d_{1}$ are sufficiently big then $(\bar{S}, \bar{I}, \bar{S}, \bar{I})_{m}$ loses its stability by a Turing bifurcation.

We illustrate the results by the following example of migration function and we are looking for conditions which imply Turing instability (diffusion driven instability).

Example 2. Trying to prepare an example comparable to that of [1], we choose $a=0.05, b=0.006, \alpha=0.06, \beta=0.0056, \gamma=0.04, c=0.05$, $m=2, n=1, d_{2}=0.05, \rho(I(t))=h \exp \left(\frac{I(t)}{h}\right)$.

The unique endemic equilibrium is $(\bar{S}, \bar{I}, \bar{S}, \bar{I})_{m}=(2.8013,7.7036,2.8013$, 7.7036). We consider $h$ as a bifurcation parameter. In this case at $h_{\text {crit }}$, we have four eigenvalues eigenvalues $\lambda_{i}(i=1,2,3,4)$ such that $\Re\left(\lambda_{i}\right)<0,(i=$ $1,2,3)$ and $\lambda_{4}=0$. Thus,

- if $h<h_{\text {crit }}$ then $\Re\left(\lambda_{i}\right)<0,(i=1,2,3,4)$ and $(\bar{S}, \bar{I}, \bar{S}, \bar{I})_{m}$ is asymptotically stable;

- if $h>h_{\text {crit }}$ then $\Re\left(\lambda_{i}\right)<0,(i=1,2,3), \lambda_{4}>0$, and $(\bar{S}, \bar{I}, \bar{S}, \bar{I})_{m}$ is unstable.

Analytical studies shown that a cross-diffusion response can stabilize an unstable equilibrium of standard system (self-diffusion) and destabilize a stable equilibrium of standard system. Numerical studies shown that if the bifurcation parameter is increased through a critical value of the bifurcation parameter $h$ the system undergoes a Turing bifurcation and the spatially homogeneous endemic equilibrium loses its stability and two new stable equilibria emerge. We conclude that the cross migration response is an important factor that should not be ignored when pattern emerges (see Figure 3). 

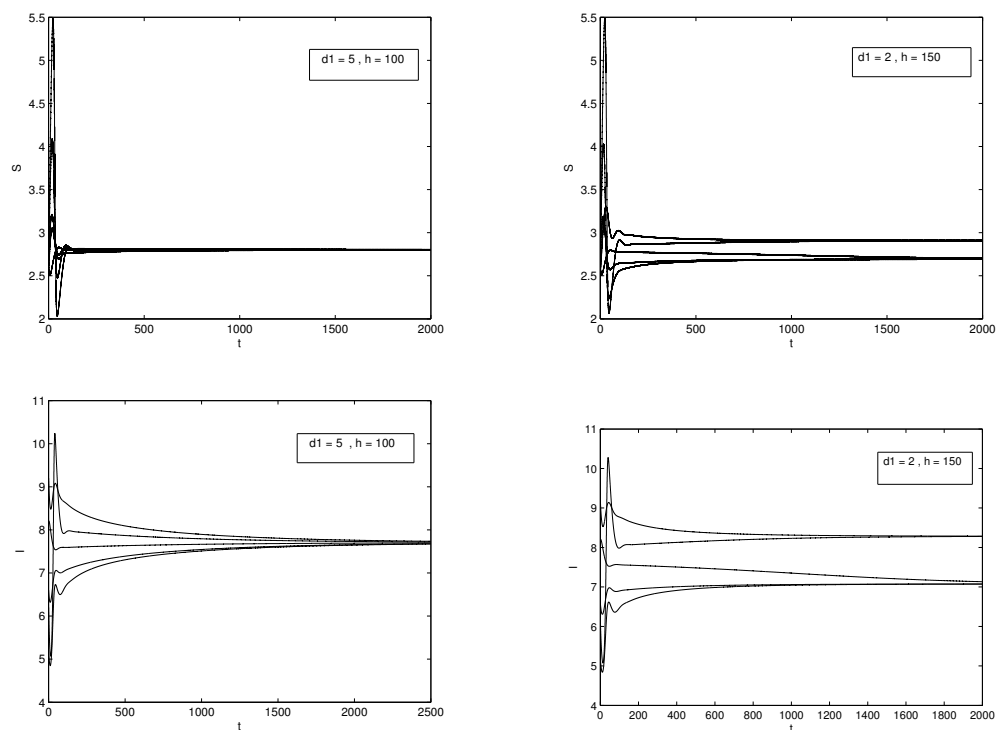

FIGURE 3. Left figures: The solutions $S$ and $I$ before bifurcation at $d_{1}=5$ and $h=100$; the unstable solution with self diffusion stable is stable with cross diffusion. Right figures: The solutions $S$ and $I$ after bifurcation at $d_{1}=2$ and $h=150$; the solutions loss its stability by Turing bifurcation. (Figure produced by applying MATLAB.)

It should be noted that, after the bifurcation, the sum of the stable equilibrium values of species $S$ at the two patches (and, similarly, that of species $I$ ) is equal to the double of its spatially homogeneous equilibrium value $\bar{S}$ (resp., $\bar{I})$.

\section{CONCLUSIONS}

In this paper, we have developed a theoretical framework for studying the phenomenon of pattern formation in a SIS (Susceptible - Infective - Susceptible) epidemic model with saturated incidence rate in order to stimulate the dynamics of disease transmission under the influence of a population migration among identical patches. Applying a stability analysis and suitable numerical simulations, we investigate the Turing parameter space and the Turing bifurcation diagram, this case is a well-known phenomenon of cross-diffusion driven instability. Numerical calculations show that a two new equilibria with non equals compounds emerge and these equilibria are asymptotically stable, so that this is a pitchfork bifurcation. 


\section{ACKNOWLEDGMENTS}

The authors extend their appreciation to the Deanship of Scientific Research at King Khalid University for funding this work though General Project under grant number (G.R.P-45-38).

\section{REFERENCES}

[1] R. M. Andereson and R. M. May, "Population biology of infectious disease: Part i," Nature, vol. 280, pp. 361-367, 1979.

[2] F. Brauer and P. Van den Driessche, "Models for transmission of disease with immigration of infectives," Math. Biosci., vol. 171, pp. 143-154, 2001.

[3] G. Carrero and M. Lizana, "Pattern formation in a sis epidemiological model," Can. Appl. Math. $Q$. , vol. 11, pp. 1-22, 2003.

[4] M. Farkas, Mathematical Models in Biology. San Diego, CA: Academic Press, 2001.

[5] S. Kovacs and S. Aly, "Stability of a delayed system modelling host-parasite association," Canadian Applied Mathematics Quarterly, vol. 18, no. 1, 2010.

[6] C. Li, C. Tsai, and S. Yang, "Analysis of epidemic spreading of an sirs model in complex heterogeneous networks," Commun. Nonlinear Sci. Numer. Simul., vol. 19, pp. 1042-1054, 2014.

[7] L. Li, Z. Jin, and G. Sun, "Spatial pattern of an epidemic model with cross-diffusion," Chin. Phys. Lett., vol. 25, pp. 3500-3503, 2009.

[8] X.-Z. Li, W.-S. Li, and M. Ghosh, "Stability and bifurcation for an sis epidemic model with treatment," Chaos Solitons Fractals, vol. 42, pp. 2822-2832, 2009.

[9] W. M. Liu, H. W. Hethcote, and S. A. Levin, "Dynamical behavior of epidemiological models with nonlinear incidence rates," J. Math. Biol., vol. 25, no. 4, pp. 359-380, 1987.

[10] J. Lou and T. Ruggeri, "The dynamics of spreading and immune strategies of sexually transmitted diseases on scale-free network," J. Math. Anal. Appl., vol. 365, pp. 210-219, 2010.

[11] J. D. Murray, Mathematical Biology I: An Introduction. Springer 3rd edition, 2007.

[12] A. Okubo and S. Levin, Diffusion and Ecological Problems. Modern Perspectives, 2001.

[13] B. Stefan and B. Ricardo, "A fully adaptive numerical approximation for a two-dimensional epidemic model with nonlinear cross-diffusion," Nonlinear Analysis: Real World Application, vol. 12, pp. 2888-2903, 2011.

[14] G. Sun, Z. Jin, L. Li, M. Haque, and L. BaiLian, "Spatial pattern in a predator-prey system with both self- and cross-diffusion," Int. J. Mod. Phy. C, vol. 20, pp. 71-84, 2009.

[15] G. Sun, Z. Jin, L. Li, M. Haque, and L. BaiLian, "Spatial patterns of a predator-prey model with cross diffusion," Nonlinear Dyn., vol. 69, pp. 1631-1638, 2012.

[16] G. Sun, Z. Jin, Q.-X. Liu, and L. Li, "Spatial pattern in an epidemic system with cross-diffusion of the susceptible," J. Biol. Sys., vol. 17, pp. 141-152, 2009.

[17] G. Sun, Z. Jin, Q. Liu, and L. Li, "Pattern formation induced by cross-diffusion in a predator-prey system," Chinese Physics B, vol. 17, no. 11, pp. 3936-3941, 2008.

[18] A. M. Turing, "The chemical basis of morphogenesis," Phil. Trans. R. Soc. London B, pp. 37-72, 1952.

[19] J. Wang and Q. Jiang, "Analysis of an sis epidemic model with treatment," Advances in Difference Equations, vol. 2014, p. 246, 2014.

[20] Y. Xiao, Y. Zhou, and S. Tang, "Modelling disease spread in dispersal networks at two levels," Math. Med. Biol., vol. 28, pp. 227-244, 2011.

[21] W. Yi, W. Ji., and Z. Li, "Cross diffusion-induced pattern in an si model," Math. Biosci.Applied Mathematics and Computation, vol. 217, pp. 1965-1970, 2010. 
[22] H. Zhang and X. Fu, "Spreading of epidemics on scale-free networks with nonlinear infectivity," Nonlinear Anal.: Theory Meth. Appl., vol. 70, pp. 3273-3278, 2009.

Authors' addresses

\section{Ali Al-Qahtani}

Current address: King Khalid University, Faculty of Science, Department of Mathematics, Applied Mathematics Group, Abha 9004, Saudi Arabia

\section{Shaban Aly}

Al-Azhar University, Faculty of Science, Mathematics Department, Assiut 71511, Egypt

Current address: King Khalid University, Faculty of Science, Department of Mathematics, Applied Mathematics Group, Abha 9004, Saudi Arabia

E-mail address: shhaly70@yahoo.com

\section{Fatma Hussien}

Department of Mathematics, Faculty of Science, Assiut University, Egypt 